\title{
Arterial Stiffening Contributes to Impairment of Cerebrovascular Reactivity in Patients With Coronary Artery Disease Without Carotid Stenosis
}

\author{
D. RUCKA ${ }^{1,2}$, J. MAREK ${ }^{1}$, Z. RUCKLOVA ${ }^{1}$, J.-C. LUBANDA ${ }^{1}$, S. HAVRANEK ${ }^{1}$, \\ J. SKVARIL ${ }^{2}$, P. VAREJKA ${ }^{1}$, M. CHOCHOLA ${ }^{1}$, D. KARETOVA ${ }^{1}$, J. KORINEK ${ }^{1,3}$, \\ A. LINHART ${ }^{1}$
}

${ }^{1}$ Second Department of Internal Medicine - Cardiology and Angiology, General University Hospital, First School of Medicine, Charles University, Prague, Czech Republic, ${ }^{2}$ Division of Cardiovascular Diseases, Military University Hospital Prague, First School of Medicine, Charles University, Prague, Czech Republic, ${ }^{3}$ International Clinical Research Center, St. Anne's University Hospital Brno, Brno, Czech Republic

Received May 29, 2014

Accepted October 8, 2014

On-line December 22, 2015

\begin{abstract}
Summary
Impaired cerebrovascular reactivity (CVR), an important risk factor for future stroke, is affected by a presence carotid stenosis. However, in some cases CVR can be impaired in the absence of carotid stenosis due to several poorly characterized mechanisms. We hypothesized that arterial stiffening as observed in coronary heart disease (CHD) could be associated with alteration in CVR in CHD patients without carotid stenosis. The study population consisted of patients referred for coronary angiography without significant carotid stenosis $(<50 \%)$. CVR was evaluated by breath holding index (BHI) measured with transcranial color code duplex ultrasound. Arterial stiffness was assessed by pulse wave velocity (PWV) measured by the oscillometric method. The extent of coronary atherosclerosis was quantified by Gensini score (GS). Out of 186 subjects, sixty-two patients fulfilled the inclusion and exclusion criteria. BHI decreased with increasing PWV $(r=-0.47, p<0.001)$. Decrease in BHI was significantly inversely associated with GS $(r=-0.61$, $p<0.001)$. GS was associated with PWV $(p<0.001)$. In conclusion, impaired CVR was associated with increased arterial stiffening in CHD patients in the absence of significant carotid stenosis. Thus, we speculate that increased arterial stiffness may at least partially contribute to the pathophysiology of CVR alteration in coronary artery disease.
\end{abstract}

\section{Key words}

Cerebrovascular reactivity - Arterial stiffness - Coronary artery disease

\section{Corresponding author}

J. Korinek, Second Department of Internal Medicine - Cardiology and Angiology, General University Hospital, First School of Medicine, Charles University, U Nemocnice 2, 12808 Prague 2, Czech Republic. Fax: +420 224912154. E-mail: jkorinek@email.cz

\section{Introduction}

The cerebral circulation maintains sufficient cerebral blood flow under various hemodynamic conditions via regional vasoconstriction and vasodilatation. Vasodilatory capacity can be characterized by cerebrovascular reactivity (CVR) (Furst et al. 1994) which can be assessed by the breath holding index (BHI) measuring the degree of vasorelaxation in response to an increased carbon dioxide level during breath hold.

Impaired CVR has been suggested as a risk factor for future stroke (Silvestrini et al. 2000) and has been implicated in the pathophysiology of multiple neurologic and psychiatric conditions including white matter hyperdensities (Marstrand et al. 2002), subcortical 
infarctions (Cupini et al. 2001), altered cognition, dementia (Silvestrini et al. 2006) and Parkinson disease (Gurevich et al. 2006). CVR can be predominantly altered in the presence of stenosis or occlusion of carotid or cerebral arteries due to hemodynamic abnormalities. In addition, it is influenced by chemical stimuli such as carbon dioxide or nitric oxide levels. Decreased CVR has been associated with aging (Matteis et al. 1998), smoking (Silvestrini et al. 1996), arterial hypertension (Ficzere et al. 1997), dyslipidemia (Wijnhoud et al. 2006) and diabetes (Fulesdi et al. 1999). However, the pathophysiology of CVR impairment without the presence of significant stenoses in the carotid or intracerebral arteries has been not entirely elucidated.

It has been recently shown that increased arterial stiffness may be associated with decreasing BHI in healthy population (Zavoreo and Demarin 2010) and in diabetic patients (Zavoreo et al. 2012). It seems plausible that increased arterial stiffening may limit vasodilatation and thus may participate on impaired CVR. Nevertheless, the relationship between arterial stiffness and CVR in coronary heart disease (CHD) has not been yet established.

We hypothesized that arterial stiffening as observed in CHD could be associated with CVR alteration in CHD patients without carotid stenosis. Therefore, we performed a study in patients referred for coronary angiography who did not have any significant carotid or cerebral artery stenosis. BHI was assessed by a transcranial color-coded duplex sonography (TCCS) of the middle cerebral artery (MCA) and arterial stiffness was evaluated by pulse wave velocity (PWV) measurements.

\section{Methods}

\section{Patient population and study design}

One hundred eighty-seven consecutive patients (all Caucasian) with suspected CHD (chronic angina, dyspnea as equivalent to angina or positive stress test) referred for coronary angiography between December 2007 and May 2008, were prospectively evaluated. Informed consent was obtained from all participants. The study was approved by the local ethics committee and complies with the Declaration of Helsinki. All patients underwent careful cardiologic and neurologic examinations the day before coronary angiography, including a detailed patient history, review of current symptoms and medical treatment, physical examination, blood tests, 12-lead electrocardiogram and transthoracic echocardiography. Carotid ultrasound and TCCS with BHI evaluation were performed the following morning prior to any drug administration and before coronary angiography. For further analysis, the study population was divided into two groups based on Gensini score (GS) results: group one (considered as controls for the purpose of the study) with GS 0 (normal coronary angiogram) and the remaining population with $\mathrm{GS} \geq 1$, i.e. patients with coronary artery disease. The study inclusion criteria included good quality TCCS on both the left and right MCA, sinus rhythm without history of atrial fibrillation or flutter, preserved left ventricular ejection fraction (>50\%), hemoglobin level $\geq 10 \mathrm{~g} / \mathrm{dl}$, peripheral oxygen saturation $>96 \%$, $\mathrm{pH}$ within physiological range on blood gas analysis, serum creatinine $<150 \mu \mathrm{mol} / 1$ and neurological examination without pathological findings. The exclusion criteria included previous coronary artery bypass graft surgery or percutaneous coronary intervention, acute or history of acute coronary syndrome or myocardial infarction, acute or history of stroke or transient ischemic attack, significant carotid stenosis or occlusion or intracranial cerebral artery stenosis identified by carotid ultrasound and TCCS, congenital heart disease, moderate or severe valvular heart disease, pericardial disease, restrictive or hypertrophic cardiomyopathy, moderate or severe restrictive or obstructive pulmonary disease, and inflammatory disease. Presence of hypertension, diabetes, hyperlipidemia and metabolic syndrome were defined according to current guidelines. Subjects were classified according to smoking habits to never smoker, past smoker or current smoker. Body mass index (BMI) was calculated in all subjects.

\section{Blood tests}

Blood samples were obtained from all subjects and analyzed in the local laboratory. Measurements included urea, creatinine, total cholesterol, LDL, HDL, triglyceride level, blood glucose, glycosylated hemoglobin $\left(\mathrm{HbA}_{1 \mathrm{c}}\right)$ levels, high sensitivity C-reactive protein (hsCRP) and hemoglobin.

\section{Transthoracic echocardiography}

Standard transthoracic echocardiography was performed using a Philips iE 33 machine. The Simpson formula was used for calculation of left ventricular ejection fraction. 


\section{Carotid ultrasound and TCCS}

Carotid ultrasound was performed with the same ultrasound equipment with a $7.5 \mathrm{MHz}$ linear probe to exclude significant common or internal carotid stenosis (>50\%, defined by maximal systolic blood flow velocity in the stenosis $>125 \mathrm{~cm} / \mathrm{s}$ ) or occlusion (Grant et al. 2003). TCCS was then performed to exclude intracranial MCA and posterior cerebral or anterior cerebral artery stenosis with the same machine using a $2 \mathrm{MHz}$ probe. The cerebral arteries were examined through the temporal window in the supine position bilaterally and the basilar artery was interrogated via the foramen magnum in the sitting position with the neck ante-flexed. The presence of stenosis $>50 \%$ was defined as the peak systolic velocity at the site of stenosis $(>220 \mathrm{~cm} / \mathrm{s}$ for the MCA, $>155 \mathrm{~cm} / \mathrm{s}$ for the anterior cerebral artery, $>145 \mathrm{~cm} / \mathrm{s}$ for the posterior cerebral artery and $>140 \mathrm{~cm} / \mathrm{s}$ for the basilar artery; Baumgartner et al. 1999) or a difference in flow rates $>30 \mathrm{~cm} / \mathrm{s}$ between both sides (Ley-Pozo and Ringelstein 1990).

\section{Cerebrovascular reactivity examination - BHI test}

CVR was evaluated by TCCS in combination with the BHI test as described previously (Markus and Harrison 1992). Briefly, the examination was performed in the supine position under baseline conditions in the morning (at least 60 min after awakening, before morning drug administration in a quiet and warm room and at least $8 \mathrm{~h}$ without drinking caffeinated beverages, eating or smoking). Subjects were instructed to take a deep breath and hold it for at least $30 \mathrm{~s}$ and practised several times to avoid the Valsalva phenomenon. Actual BHI evaluation was performed twice for each side with at least two minutes rest between measurements (Silvestrini et al. 2009). A mean of 3 consecutive cycles of MCA velocities at rest just prior to and at the peak of the breath holding phase were taken for instantenous calculation of BHI and the average of four measurements in each patient was used for further analysis. Heart rate and blood pressure were measured prior to and at the end of each breath hold (OMRON M5 Professional, Omron Healthcare Co. Ltd, Japan) and the duration of breath holding was recorded.

BHI was calculated using the formula: $\mathrm{BHI}=$ $[100 *(\mathrm{Vbh}-\mathrm{Vr}) / \mathrm{Vr}] / \mathrm{S}$ where $\mathrm{Vbh}$ was the mean MCA velocity at the end of breath holding, $\mathrm{Vr}$ was the MCA mean velocity at rest and $\mathrm{S}$ was the duration of breath holding in seconds.

\section{Coronary angiography}

Coronary angiograms were performed on GE Inova 2100 equipment and interpreted visually in two orthogonal views by skilled observers who were blinded to TCCS results. The Gensini score, which was used in other studies (Adams et al. 1995) and provides information about both the extent and severity of coronary atherosclerosis, was evaluated in each patient as described previously. In brief, the coronary tree was divided into 8 segments (left main, left anterior descending, left circumflex, left diagonal, left marginal, right, right posterior descending and right marginal arteries). The most severe stenosis of each coronary segment was graded from 0 to 4 according the degree of atherosclerotic involvement (grade 0: no stenosis, grade 1: $1-49 \%$ stenosis, grade 2: 50-74\% stenosis, grade 3: 75-99\% stenosis, grade 4: $100 \%$, i.e. occlusion) giving a total score between 0 and 32 .

\section{Arterial stiffness evaluation}

Aortic pulse wave velocity (PWV) as a parameter of arterial stiffness was determined as described previously in detail elsewhere by a validated (Horvath et al. 2010) arteriograph device (TensioMed Kft., Budapest, Hungary). Mesurements were performed in concordance with international guidelines in a room with a constant temperature $\left(20^{\circ} \mathrm{C}\right)$ where patients were not affected by any external environmental influences. The data analysis was performed by the device software and the PWV (m/s) was calculated as (Jug-Sym)/(RT/2) where Jug-Sym means a distance from sternal notch to the upper edge of the pubic bone (Jugulum - Symphysis) and RT denotes the time interval measured by the device between the peaks of the direct and reflected systolic wave (return time) (Horvath et al. 2010).

\section{Statistical analysis}

The results are expressed as mean \pm standard deviation for continuous variables and relative frequencies for binary and categorical variables. The analyses were performed using Statistica 6.1 (StatSoft, Inc., Tulsa, OK, USA) and R 3.0 (R Foundation for Statistical Computing, Vienna, Austria). The Wilcoxon test was used for comparisons of two groups. The Chisquare test was used for categorical data and to compare proportions. Pearson correlation coefficient was used to quantify correlation, except for correlations with GS, where Spearman's rank correlation coefficient was used. Univariate linear regression was employed to analyze 
relationships between $\mathrm{BHI}$ and other variables. BHI was logarithmically transformed for linear regression modeling purposes. The F-test was used to test significance of categorical covariates with $>2$ categories. Multivariate linear regression was used to correct for confounders (variables significantly predictive in univariate models) when evaluating the relationship between BHI and PWV and to evaluate independent predictors of $\mathrm{BHI} ; \mathrm{p}<0.05$ values were considered statistically significant.

\section{Results}

\section{Study population}

Out of the 187 consecutive subjects, 62 patients fullfilled the inclusion and exclusion criteria. Thirty-nine subjects were excluded due to poor temporal windows or suboptimal quality of TCCS, 28 due to atrial fibrilation or flutter, 21 due to previous PCI or CABG, 11 due to systolic dysfunction, 6 for history of stroke, TIA or head trauma, 5 for lung disease, 4 for carotid stenosis, 4 for insuficient ability of breathholding, 3 for moderate to severe valvular disease, 2 for known sleep apnea on treatment, 1 for restlessness and 1 for hypertensive crisis just prior to scheduled TCCS.

\section{Study population characteristics}

The clinical profile including hemodynamic parameters, blood tests and medications, for the patient groups divided according to the $\mathrm{GS}$ ( $\mathrm{GS}=0$ considered as controls) is presented in Table 1. Patients with coronary atherosclerosis (GS $\geq 1$ ) were older with higher systolic blood pressure, were more likely to have diabetes mellitus with higher fasting blood glucose, and had higher hsCRP. They also tended to have higher LDL, and were more frequently treated with aspirin, betablockers and statins.

Cerebrovascular reactivity, arterial stiffness and coronary atherosclerosis

As compared to controls, patients with GS $\geq 1$ had a significantly lower BHI and higher PWV. BHI was significantly inversely correlated with PWV ( $\mathrm{r}=-0.47$, $\mathrm{p}<0.001$, Fig. 1). Furthermore, BHI significantly decreased with increasing GS $(r=-0.61, p<0.001)$. In addition, BHI was inversely associated with age, systolic blood pressure, hsCRP and diabetes (Table 2). There was no significant association between $\mathrm{BHI}$ and other parameters including gender, obesity, BMI, serum creatinine, lipids (total cholesterol, LDL- and HDL- cholesterol, triglycerides). After correction for the above mentioned baseline confounding variables, the only parameters that remained significantly associated with BHI were PWV and GS.

In univariate analysis, PWV correlated with GS. Additionally, PWV were associated with age, systolic blood pressure, hsCRP, diabetes and dyslipidemia (Table 3).

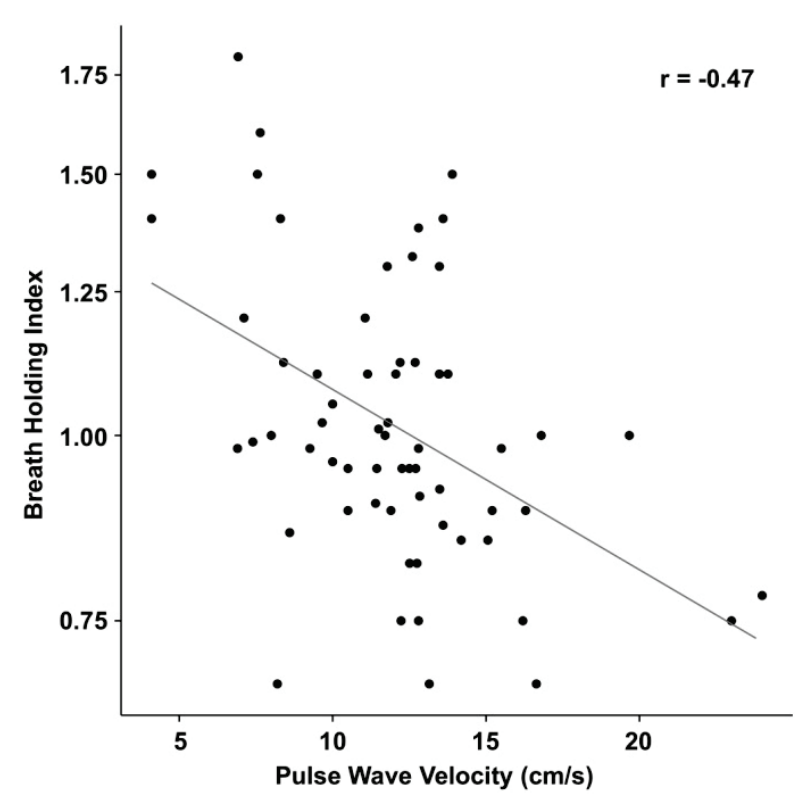

Fig. 1. The inverse relationship of log transformed breath holding index and pulse wave velocity.

\section{Discussion}

In the present study, we demonstrate for the first time an association between impaired cerebrovascular reactivity evaluated by breath holding index and arterial stiffness characterized by pulse wave velocity in patients referred for coronary angiography who are free of significant carotid artery stenosis. This relationship was at least in part independent of other confounding variables including age, atherosclerosis risk factors and even the extent of coronary atherosclerosis as assessed by GS.

In addition, our study shows that association of CVR and coronary atherosclerosis evaluated by the Gensini score exists even in absence of carotid artery stenosis. Since both GS and CVR are related to PWV, arterial stiffness may be one of the missing links between coronary and cerebrovascular manifestations. 
Table 1. Baseline characteristics of patient subgroups according to the Gensini score.

\begin{tabular}{|c|c|c|c|}
\hline Variable & GS $0(N=12)$ & $G S \geq 1(N=50)$ & p-value \\
\hline Gensini score & $0 \pm 0$ & $6.1 \pm 4.3$ & $<0.001$ \\
\hline Male, $n(\%)$ & $7(58 \%)$ & $32(64 \%)$ & 0.715 \\
\hline Age, years & $57 \pm 8$ & $67 \pm 10$ & $<0.001$ \\
\hline Body mass index, $\mathrm{kg} / \mathrm{m}^{2}$ & $28.4 \pm 2.0$ & $27.7 \pm 4.6$ & 0.432 \\
\hline Smoking & & & 0.91 \\
\hline Never smokers, $n(\%)$ & $5(42 \%)$ & $22(44 \%)$ & - \\
\hline Past smokers, $n(\%)$ & $2(17 \%)$ & $6(12 \%)$ & - \\
\hline Active smokers, $n(\%)$ & $5(42 \%)$ & $22(44 \%)$ & - \\
\hline Diabetes mellitus, $n(\%)$ & $0(0 \%)$ & $19(38 \%)$ & 0.01 \\
\hline Hypertension, $n(\%)$ & $10(83 \%)$ & $41(82 \%)$ & 0.140 \\
\hline Systolic BP, mm Hg & $136 \pm 15$ & $151 \pm 19$ & 0.021 \\
\hline Diastolic BP, $\mathrm{mm} \mathrm{Hg}$ & $76 \pm 11$ & $82 \pm 10$ & 0.111 \\
\hline Heart rate, beats/min & $67 \pm 11$ & $70 \pm 10$ & 0.305 \\
\hline Dyslipidemia, $n(\%)$ & $7(58 \%)$ & $39(78 \%)$ & 0.162 \\
\hline Total cholesterol, $\mathrm{mmol} / \mathrm{l}$ & $5.0 \pm 0.9$ & $5.2 \pm 0.9$ & 0.318 \\
\hline LDL cholesterol, mmol/l & $2.8 \pm 0.7$ & $3.2 \pm 0.8$ & 0.086 \\
\hline HDL cholesterol, mmol/l & $1.2 \pm 0.2$ & $1.2 \pm 0.3$ & 0.407 \\
\hline Triglycerides, $\mathrm{mmol} / \mathrm{l}$ & $2.1 \pm 0.9$ & $2.0 \pm 0.9$ & 0.653 \\
\hline Fasting glucose, $\mathrm{mmol} / \mathrm{l}$ & $4.9 \pm 0.7$ & $6.0 \pm 2.0$ & 0.113 \\
\hline$H b A_{l c}, \%$ & $4.8 \pm 0.7$ & $5.4 \pm 1.7$ & 0.277 \\
\hline Metabolic syndrome, $n(\%)$ & $7(58 \%)$ & $30(60 \%)$ & 0.92 \\
\hline Serum creatinine, $\mu \mathrm{mol} / \mathrm{l}$ & $110 \pm 7$ & $114 \pm 9$ & 0.135 \\
\hline Hemoglobin $\mathrm{g} / \mathrm{dl}$ & $12.0 \pm 0.8$ & $12.6 \pm 1.2$ & 0.092 \\
\hline$h s C R P, m g / l$ & $2.3 \pm 1.1$ & $3.9 \pm 1.7$ & 0.002 \\
\hline$A C E I / A R B S, n(\%)$ & $9(75 \%)$ & $35(70 \%)$ & 0.732 \\
\hline Betablockers, $n(\%)$ & $1(8 \%)$ & $19(38 \%)$ & 0.048 \\
\hline Calcium channel blockers, $n(\%)$ & $5(42 \%)$ & $26(52 \%)$ & 0.52 \\
\hline Diuretics, $n(\%)$ & $3(25 \%)$ & $10(20 \%)$ & 0.702 \\
\hline Aspirin, $n(\%)$ & $5(42 \%)$ & $39(78 \%)$ & 0.013 \\
\hline Statins, $n(\%)$ & $3(25 \%)$ & $32(64 \%)$ & 0.014 \\
\hline Oral hypoglycemic agents, $n(\%)$ & $0(0 \%)$ & $4(8 \%)$ & 0.311 \\
\hline Insulin treatment, $n(\%)$ & $0(0 \%)$ & $10(20 \%)$ & 0.091 \\
\hline Breath Holding Index & $1.35 \pm 0.27$ & $0.97 \pm 0.17$ & $<0.001$ \\
\hline Pulse Wave Velocity $(\mathrm{m} / \mathrm{s})$ & $9.2 \pm 3.3$ & $12.7 \pm 3.5$ & 0.004 \\
\hline
\end{tabular}

ACEI, angiotensin-converting-enzyme inhibitors; ARBs, angiotensin II receptor blockers; BP, blood pressure; HbA 1 , glycosylated hemoglobin; hsCRP, high sensitivity C-reactive protein.

\section{Cerebrovascular reactivity and arterial stiffness}

Cerebrovascular flow and reactivity are regulated by a complex of metabolic, myogenic and neurogenic mechanisms as well as by passive properties of the blood vessels (Ainslie and Duffin 2009) including arterial stiffness. Increased rigidity of the arterial wall limits its capacity for vasodilatation in response to physiologic stimuli. Arterial stiffening increases with age and is known to be promoted by several factors including hypertension, diabetes, chronic kidney disease, i.e. by almost all stimuli leading to atherosclerosis (Zieman et al. 2005). Interestingly, only two studies have investigated the relationship between arterial stiffening and CVR, one was performed in a healthy population and the second one in diabetic patients. The first study found a very good inverse correlation between BHI and arterial stiffness 
Table 2. Univariate and multivariate linear regression analysis of $\mathrm{BHI}$ and other variables.

\begin{tabular}{|c|c|c|c|c|c|c|c|c|c|c|}
\hline \multirow{3}{*}{ Variable } & \multicolumn{5}{|c|}{ Univariate } & \multicolumn{5}{|c|}{ Multivariate } \\
\hline & & & $2.5 \%$ & $97.5 \%$ & & & & $2.5 \%$ & $97.5 \%$ & \\
\hline & $\beta$ & $\mathbf{S E}$ & & & p-value & $\boldsymbol{\beta}$ & $\mathbf{S E}$ & CI & $\mathrm{Cl}$ & p-value \\
\hline Age (per 10 years) & -0.08 & 0.02 & -0.13 & -0.03 & 0.003 & & & & & 0.38 \\
\hline $\begin{array}{l}\text { Systolic BP } \\
\text { (per } 10 \mathrm{~mm} \mathrm{Hg} \text { ) }\end{array}$ & 0.04 & 0.01 & -0.07 & -0.01 & 0.006 & & & & & 0.320 \\
\hline hsCRP (per mg/l) & -0.03 & 0.02 & -0.06 & 0.00 & 0.046 & & & & & 0.375 \\
\hline Diabetes & -0.17 & 0.06 & -0.28 & -0.05 & 0.005 & & & & & 0.334 \\
\hline Pulse Wave Velocity & -0.03 & 0.01 & -0.04 & -0.01 & $<0.001$ & -0.02 & 0.01 & -0.03 & -0.00 & 0.022 \\
\hline Gensini score $\geq 1$ & -0.32 & 0.06 & -0.44 & -0.21 & $<0.001$ & -0.22 & 0.07 & -0.36 & -0.09 & 0.002 \\
\hline
\end{tabular}

$\mathrm{BHI}$, breath holding index; $\mathrm{BP}$, blood pressure; $\mathrm{CI}$, confidence interval; hsCRP, high sensitivity C-reactive protein; $\mathrm{SE}$, standard error.

Table 3. Predictors of PWV.

\begin{tabular}{lccccc}
\hline \multicolumn{1}{c}{ Variable } & $\boldsymbol{\beta}$ & SE & $\mathbf{2 . 5 \%} \mathbf{C I}$ & $\mathbf{9 7 . 5 \%} \mathbf{C I}$ & p-value \\
\hline hSCRP & 1.14 & 0.23 & 0.68 & 1.61 & $<0.001$ \\
HbA lc $_{\text {Hyperlipidemia }}$ & 0.91 & 0.28 & 0.35 & 1.46 & 0.002 \\
Diabetes status & 2.51 & 1.03 & 0.49 & 4.52 & 0.018 \\
Gensini Score $\geq 1$ & 2.64 & 0.97 & 0.70 & 4.57 & 0.008 \\
& 3.51 & 1.11 & 1.35 & 5.68 & 0.002 \\
\hline
\end{tabular}

$\mathrm{CI}$, confidence interval; $\mathrm{HbA}_{1 \mathrm{c}}$, glycosylated hemoglobin; hsCRP, high sensitivity C-reactive protein; PWV, pulse wave velocity; $\mathrm{SE}$, standard error.

(Zavoreo and Demarin 2010) and concluded that in a healthy population the relationship was a function of aging. The second study was performed in diabetic patients and similar trends were shown. The BHI was decreasing with increasing arterial stiffness in diabetics, more so in patients with poor glycemic control. However, the exact correlation coefficient was not provided (Zavoreo et al. 2012). In our study, we have shown that BHI was moderately correlated $(r=-0.47)$ with PWV, therefore decreased CVR can be attributed to arterial stiffening only in part. On the other hand, this moderate association indicates that other factors are likely be involved in CVR alteration in coronary artery disease patients. Along with arterial stiffness, impaired CVR has been previously shown to be associated with aging (Matteis et al. 1998), blood pressure (Ficzere et al. 1997, Claassen et al. 2009), dyslipidemia (Wijnhoud et al. 2006) and diabetes (Fulesdi et al. 1999). In concordance with those studies, we observed that BHI decreased with age, systolic blood pressure and in diabetics.
Cerebrovascular reactivity and carotid and cerebral artery stenoses

Atherosclerosis, apart from endothelial dysfunction with altered nitric oxide production and increased wall stiffness in plaque area, can significantly participate in the alteration of CVR by proximal vascular narrowing. A significant carotid stenosis or occlusion is considered as one of the most important factors affecting CVR (Furst et al. 1994). The stenotic lesion may lead to a post-stenotic compensatory arterial vasodilatation, thus constraining further vasodilatation capacity and subsequently leading to a CVR decrease. The exhausted vasodilatation reserve could be the main mechanism involved in the higher risk of strokes reported in patients with carotid stenosis or occlusion who have decreased CVR compared to those without (Gupta et al. 2012). The mechanism by which significant intracerebral arterial stenosis negatively affects CVR is probably similar (Chen et al. 2014). In our study, patients with either carotid or cerebral artery stenoses were excluded, therefore, contribution by other mechanisms can be 
assumed. Although impaired CVR has been suggested as a risk factor for future stroke (Silvestrini et al. 2000) and patients with coronary artery disease are at increased risk of ischemic strokes, further studies are needed to evaluate predictive power of CVR in this patient population.

\section{Cerebrovascular reactivity and coronary artery disease}

The association between CVR and degree of coronary atherosclerosis has been suggested by a recent study that used transcranial Doppler ultrasound of the posterior cerebral arteries and visually evoked flow responses as a CVR marker (Rosengarten et al. 2009). However, the studied population also included patients with significant carotid occlusive disease, thus the possibility that the observed association between $\mathrm{BHI}$ and GS were due to carotid stenosis could not be ruled out.

We have observed the association between CVR and GS even in the absence of significant carotid stenoses, expanding on the results of the study performed by Rosengarten and colleagues (2009). Based on our results, we suggest that CVR may be independently related to the degree of coronary atherosclerosis. This finding would further support the presumed link between coronary atherosclerosis development and cerebrovascular functional impairment (Targonski et al. 2003).

The inverse association between BHI and hsCRP observed in our study may also reflect the overall atherosclerotic burden with associated endothelial dysfunction (Elkind 2006) and functional vascular impairment in our patients.

Other factors with a possible effect on cerebrovascular reactivity

CVR can be also modified by gender (Matteis et al. 1998) and smoking (Silvestrini et al. 1996), an association not observed in our study. The expected effect of gender could be masked by the presence of diabetes with a potentially stronger negative effect on CVR (Fulesdi et al. 2001). Additionally, most of the women involved in our study were postmenopausal who are known to have a lower BHI than premenopausal women or younger men (Matteis et al. 1998).

Cigarette smoking has a negative impact on cerebrovascular circulation and has virtually an immediate negative effect on CVR (Silvestrini et al. 1996). Interestingly in that study, no significant difference in CVR was found between controls and smokers at baseline before they smoked their first morning cigarette (Silvestrini et al. 1996). In our study, smokers were requested not to smoke from the evening before until the CVR testing was done the next morning, which could explain the absence of association between smoking and BHI in our study.

\section{Limitations}

In order to limit the effect of other hemodynamic factors on the studied relationship between CVR and arterial stiffness, we excluded patients with heart failure or low ejection fraction, history of myocardial infarction, severe valvular disease, history of stroke, presence of intracranial arterial stenosis or severe uncontrolled hypertension. This approach eliminates possible confounders and simplifies the data analysis, but on the other hand, our results are applicable only to a subset of the CHD population. Because of the cross sectional nature of the study, we cannot prove causality. The association between CRV and PWV may be due to common risk factors as well. Another limitation of the study is a relatively small study size.

Furthermore, in our study, we did not measure specifically cerebral arterial stiffness. Additional studies are required to elucidate the relationship between cerebral and systemic arterial stiffness.

Endothelial dysfunction was not evaluated in our study; however, other studies already described an association between endothelial dysfunction and impaired cerebrovascular reactivity (Lavi et al. 2006).

\section{Conclusions}

Increased arterial stiffness contributes to the impairment of cerebrovascular reactivity observed in patients with coronary artery disease independently of the presence of significant carotid stenosis. Moreover, altered CVR was associated with the degree of coronary atherosclerosis. Further studies are needed to define the role of other factors that may contribute to CVR impairment in coronary artery disease.

\section{Conflict of Interest}

There is no conflict of interest.

\section{Acknowledgements}

The study was supported by PRVOUK-P35/LF1/5, European Regional Development Fund - Project FNUSAICRC (No. CZ.1.05/1.1.00/02.0123) and project from OP Prague Competitiveness, reg. no. CZ.2.16/3.1.00/24012. We thank to TensioMed Kft., Budapest, Hungary for providing the Arteriograph device for the purpose of the study. 


\section{References}

ADAMS MR, NAKAGOMI A, KEECH A, ROBINSON J, MCCREDIE R, BAILEY BP, FREEDMAN SB, CELERMAJER DS: Carotid intima-media thickness is only weakly correlated with the extent and severity of coronary artery disease. Circulation 92: 2127-2134, 1995.

AINSLIE PN, DUFFIN J: Integration of cerebrovascular CO2 reactivity and chemoreflex control of breathing: mechanisms of regulation, measurement, and interpretation. Am J Physiol Regul Integr Comp Physiol 296: R1473-R1495, 2009.

BAUMGARTNER RW, MATTLE HP, SCHROTH G: Assessment of $>/=50 \%$ and $<50 \%$ intracranial stenoses by transcranial color-coded duplex sonography. Stroke 30: 87-92, 1999.

CHEN J, LIU J, XU WH, XU R, HOU B, CUI LY, GAO S: Impaired dynamic cerebral autoregulation and cerebrovascular reactivity in middle cerebral artery stenosis. PLoS One 9: e88232, 2014.

CLAASSEN JA, LEVINE BD, ZHANG R: Cerebral vasomotor reactivity before and after blood pressure reduction in hypertensive patients. Am J Hypertens 22: 384-391, 2009.

CUPINI LM, DIOMEDI M, PLACIDI F, SILVESTRINI M, GIACOMINI P: Cerebrovascular reactivity and subcortical infarctions. Arch Neurol 58: 577-581, 2001.

ELKIND MS: Inflammation, atherosclerosis, and stroke. Neurologist 12: 140-148, 2006.

FICZERE A, VALIKOVICS A, FULESDI B, JUHASZ A, CZURIGA I, CSIBA L: Cerebrovascular reactivity in hypertensive patients: a transcranial Doppler study. J Clin Ultrasound 25: 383-389, 1997.

FULESDI B, LIMBURG M, BERECZKI D, KAPLAR M, MOLNAR C, KAPPELMAYER J, NEUWIRTH G, CSIBA L: Cerebrovascular reactivity and reserve capacity in type II diabetes mellitus. J Diabetes Complications 13: 191-199, 1999.

FULESDI B, LIMBURG M, OLAH L, BERECZKI D, CSIBA L, KOLLAR J: Lack of gender difference in acetazolamide-induced cerebral vasomotor reactivity in patients suffering from type-1 diabetes mellitus. Acta Diabetol 38: 107-112, 2001.

FURST H, HARTL WH, JANSSEN I: Patterns of cerebrovascular reactivity in patients with unilateral asymptomatic carotid artery stenosis. Stroke 25: 1193-1200, 1994.

GRANT EG, BENSON CB, MONETA GL, ALEXANDROV AV, BAKER JD, BLUTH EI, CARROLL BA, ELIASZIW M, GOCKE J, HERTZBERG BS, KATANICK S, NEEDLEMAN L, PELLERITO J, POLAK JF, RHOLL KS, WOOSTER DL, ZIERLER RE: Carotid artery stenosis: gray-scale and Doppler US diagnosis Society of Radiologists in Ultrasound Consensus Conference. Radiology 229: 340-346, 2003.

GUPTA A, CHAZEN JL, HARTMAN M, DELGADO D, ANUMULA N, SHAO H, MAZUMDAR M, SEGAL AZ, KAMEL H, LEIFER D, SANELLI PC: Cerebrovascular reserve and stroke risk in patients with carotid stenosis or occlusion: a systematic review and meta-analysis. Stroke 43: 2884-2891, 2012.

GUREVICH T, GUR AY, BORNSTEIN NM, GILADI N, KORCZYN AD: Cerebral vasomotor reactivity in Parkinson's disease, multiple system atrophy and pure autonomic failure. J Neurol Sci 243: 57-60, 2006.

HORVATH IG, NEMETH A, LENKEY Z, ALESSANDRI N, TUFANO F, KIS P, GASZNER B, CZIRAKI A: Invasive validation of a new oscillometric device (Arteriograph) for measuring augmentation index, central blood pressure and aortic pulse wave velocity. J Hypertens 28: 2068-2075, 2010.

LAVI S, GAITINI D, MILLOUL V, JACOB G: Impaired cerebral $\mathrm{CO}_{2}$ vasoreactivity: association with endothelial dysfunction. Am J Physiol Heart Circ Physiol 291: H1856-H1861, 2006.

LEY-POZO J, RINGELSTEIN EB: Noninvasive detection of occlusive disease of the carotid siphon and middle cerebral artery. Ann Neurol 28: 640-647, 1990.

MARKUS HS, HARRISON MJ: Estimation of cerebrovascular reactivity using transcranial Doppler, including the use of breath-holding as the vasodilatory stimulus. Stroke 23: 668-673, 1992.

MARSTRAND JR, GARDE E, ROSTRUP E, RING P, ROSENBAUM S, MORTENSEN EL, LARSSON HB: Cerebral perfusion and cerebrovascular reactivity are reduced in white matter hyperintensities. Stroke 33: 972-976, 2002.

MATTEIS M, TROISI E, MONALDO BC, CALTAGIRONE C, SILVESTRINI M: Age and sex differences in cerebral hemodynamics: a transcranial Doppler study. Stroke 29: 963-967, 1998. 
ROSENGARTEN B, GREBE M, MULLER A, VOSS RK, KAPS M: Severity of coronary artery disease but not degree of coronary stenosis is correlated to cerebrovascular reactivity. Cerebrovasc Dis 28: 290-297, 2009.

SILVESTRINI M, TROISI E, MATTEIS M, CUPINI LM, BERNARDI G: Effect of smoking on cerebrovascular reactivity. J Cereb Blood Flow Metab 16: 746-749, 1996.

SILVESTRINI M, VERNIERI F, PASQUALETTI P, MATTEIS M, PASSARELLI F, TROISI E, CALTAGIRONE C: Impaired cerebral vasoreactivity and risk of stroke in patients with asymptomatic carotid artery stenosis. JAMA 283: 2122-2127, 2000.

SILVESTRINI M, PASQUALETTI P, BARUFFALDI R, BARTOLINI M, HANDOUK Y, MATTEIS M, MOFFA F, PROVINCIALI L, VERNIERI F: Cerebrovascular reactivity and cognitive decline in patients with Alzheimer disease. Stroke 37: 1010-1015, 2006.

SILVESTRINI M, PAOLINO I, VERNIERI F, PEDONE C, BARUFFALDI R, GOBBI B, CAGNETTI C, PROVINCIALI L, BARTOLINI M: Cerebral hemodynamics and cognitive performance in patients with asymptomatic carotid stenosis. Neurology 72: 1062-1068, 2009.

TARGONSKI PV, BONETTI PO, PUMPER GM, HIGANO ST, HOLMES DR JR, LERMAN A: Coronary endothelial dysfunction is associated with an increased risk of cerebrovascular events. Circulation 107: 2805-2809, 2003.

WIJNHOUD AD, KOUDSTAAL PJ, DIPPEL DW: Relationships of transcranial blood flow Doppler parameters with major vascular risk factors: TCD study in patients with a recent TIA or nondisabling ischemic stroke. $J$ Clin Ultrasound 34: 70-76, 2006.

ZAVOREO I, DEMARIN V: Breath holding index and arterial stiffness as markers of vascular aging. Curr Aging Sci 3: 67-70, 2010.

ZAVOREO I, KES VB, CORIC L, DEMARIN I: Breath holding index and arterial stiffness in evaluation of stroke risk in diabetic patients. Perspect Med 1: 156-159, 2012.

ZIEMAN SJ, MELENOVSKY V, KASS DA: Mechanisms, pathophysiology, and therapy of arterial stiffness. Arterioscler Thromb Vasc Biol 25: 932-943, 2005. 